$500 \mathrm{ccm}$ absol. Methanol zur Verseifung $3^{1} / 2$ Stdn. bei Zimmertemperatur stehen gelassen, dann mit Wasser verdünnt und mit Essigsäure schwach angesäuert. Mit Äther konnten der wäßr. Phase $124 \mathrm{~g}$ des Reaktionsgutes entzogen werden, die mit $130 \mathrm{~g}$ Phthalsäureanhydrid in $500 \mathrm{ccm}$ Pyridin in üblicher Weise zur Abtrennung der primären Alkohole behandelt wurden. Aus $21 \mathrm{~g}$ der rohen Alkohole ließen sich $6 \mathrm{~g}$ Geraniol vom Sdp.14 $100-112-115^{\circ}$ herausdestillieren.

$$
n_{\mathrm{D}}^{20} 1,4726, \quad d_{4}^{20} 0,8858 .
$$

Mol.-Refr. $\mathrm{C}_{10} \mathrm{H}_{18} \mathrm{O}$ F2, ber. 48,97, gef. 48,78.

3.5-Dinitrobenzoat: Schmp. 60 bis $61^{\circ}$ (K of le r). Die Mischprobe mit dem Dinitrobenzoat aus natürlichem Geraniol schmolz bei 60 bis $61,5^{\circ}$.

Aus einem Ansatz von $30 \mathrm{~g}$ Isopren mit 2,5 g $p$ Toluolsulfonsäure in $125 \mathrm{~g}$ Eisessig (98 bis 100\%, p.a.), also $2 \%$ des Eisessigs, der $52 \mathrm{Stdn}$. bei $18^{\circ}$ gestanden hatte, wurden $7,3 \mathrm{~g}$ Prenylacetat vom Sdp.11 46 bis $48^{\circ}$ gewonnen. Diese Fraktion, mit methanol. $\mathrm{KOH}$ verseift, ergab $3 \mathrm{~g}$ Prenol vom Sdp.751 138 bis $140^{\circ}$.

$$
n_{\mathrm{D}}^{21} 1,4450 . \quad d_{4}^{21} 0,8691 .
$$

Mol.-Refr. $\mathrm{C}_{5} \mathrm{H}_{10} \mathrm{O}$ F1, ber. 26,36, gef. 26,36 .

Phenylurethan: Schmp. $64^{\circ}$ (Kofler), Mischprobe ebenso.

Die nachfolgende Übersicht enthält die Ausbeuten aus je $30 \mathrm{~g}$ Isopren in $125 \mathrm{~g}$ Eisessig (98 bis 100proz.) mit variierten Katalysatorkonzentrationen (bezogen

\begin{tabular}{|c|c|c|c|c|c|}
\hline \multirow{2}{*}{$\begin{array}{l}p \text {-Toluol- } \\
\text { sulfon- } \\
\text { säurekon- } \\
\text { zentration } \\
\quad \text { in } \%\end{array}$} & \multirow{2}{*}{$\begin{array}{l}\text { Reakt.- } \\
\text { dauer } \\
\text { in Stdn. }\end{array}$} & \multirow{2}{*}{ 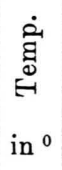 } & \multirow{2}{*}{$\begin{array}{l}\text { Gesamt- } \\
\text { ausb. roh } \\
\text { in } g\end{array}$} & \multicolumn{2}{|c|}{$\operatorname{Sdp}_{11}$} \\
\hline & & & & $\begin{array}{c}45-50^{\circ} \\
\mathrm{g}\end{array}$ & $\begin{array}{c}100-120^{\circ} \\
\mathrm{g}\end{array}$ \\
\hline 0,5 & 112 & 18 & 13 & 6,1 & 4,7 \\
\hline 1 & 18,5 & 20 & 10 & 3,5 & 3 \\
\hline 2 & 52 & 18 & 18 & 7,3 & $8, \tilde{5}$ \\
\hline 4 & 50 & 18 & 30 & 5,9 & 14 \\
\hline 5 & 48 & 18 & 33 & 4,4 & 15,9 \\
\hline 6 & 40 & 18 & 35 & 3,2 & 16,9 \\
\hline
\end{tabular}
auf Eisessig):

Durch Steigerung der $p$-Toluolsulfonsäurekonzentration kann also die Gesamtausbeute an Polymerisat erhöht werden, doch nimmt die Ausbeute an Prenylacetat parallel damit wesentlich ab, zugunsten der höher siedenden Fraktionen, die dann zum größten Teil aus Kohlenwasserstoffen bestehen.

Bei Versuchen mit $1 \%$ Trichloressigsäure $(k=$ $\left.2 \cdot 10^{-1 / 18^{\circ}}\right)$ oder $1 \%$ Pikrinsäure $\left(k=1,6 \cdot 10^{-1} / 25^{\circ}\right)$ in Eisessig konnte keine Anlagerung oder Polymerisation beobachtet werden.

Hr. Hans Lieber hat mich bei der Ausführung der Versuche fleißig unterstützt.

\title{
Weitere Röntgeninterferenzen bei Keratinen ${ }^{1}$
}

\author{
Von Helmut $Z_{\text {AhN }}$
}

\author{
Aus dem Institut für Textilchemie in Badenweiler
}

(Z. Naturforschg. 1, 690-691 [1946]; eingegangen am 24. Oktober 1946)

\begin{abstract}
Keratin-Röntgenogramme enthalten verwaschene D e b y e - S c h e r r e r - Ringe, deren Schwärzungsmaxima Abständen von $4 \div 4,6,2,1 \div 2,3,1,5 \div 1,6$ und $1,1 \div 1,2 \AA$ entsprechen.
\end{abstract}

$\mathrm{D}$ ie Röntgenogramme von Keratinen enthalten im normalen Winkelbereich außer den von W. T. Astbury und A. Street ${ }^{2}$ festgestellten Interferenzen noch einige verwaschene DebyeScherrer-Ringe, welche eigenartigerweise bisher übersehen worden sind. Sie entsprechen Netzebenenabständen von $4 \div 4,6^{3}, 2,1 \div 2,3,1,5 \div 1,6$ und 1,1 - 1,2 A. Ein Teil dieser Interferenzen wird bei verhältnismäßig großen Winkeln abgebeugt und entzieht sich dem Nachweis in der bei Faserauf-

1 20. Mitteilung aus der Reihe ,Struktur und Reaktionsfähigkeit der Keratine", vorige Mitteilung vergl. E. El öd u. H. Z a h n, Melliand Textilber. 27, 68 [1946].

2 Philos. Trans. Roy. Soc. London Ser. A 230, 75 [1931]. nahmen üblichen L a u e-Kammer, während eine zylindrische Aufnahmekamera günstigere Intensitätsverhältnisse bietet. Die Interferenzen treten bei verschiedenen Keratinsorten, wie Wolle, Roßhaaren, Stachelschweinkiel, auf, und zwar ist es in erster Näherung gleichgültig, ob die Proben im ungedehnten ( $\alpha$-Keratin), im gedehnten ( $\beta$-Keratin) oder denaturierten Zustande (d-Keratin) vorliegen. Bezüglich der Anisotropieverhältnisse fällt auf, daß die Interferenzen im Äquator etwas stärker ausgeprägt sind, man hat sie jedoch als Inter-

3 R. B. Cor e y u. R. W. G. W y c k of f (Journ. Biol. Chem. 114, 407 [1936]) verzeichneten ebenfalls einen amorphen Halo auf dem Äquator bei $4,6 \AA$. 
ferenzringe, welche verwaschen und diffus auftreten, anzusprechen. Die Intensitäten nehmen mit steigendem Abbeugungswinkel stark ab.

Zweifelsohne fallen die Interferenzen bei 1,6 und 1,1 $\AA$ mit keiner bekannten Keratinlinie zusammen, hingegen ist eine Koinzidenz von $2,1 \AA$ mit (030) und (230) der dritten Schichtlinie des $\beta$-Keratins denkbar. Da aber diese Interferenzen des $\beta$-Keratins in Form von orientierten Sicheln bzw. Interferenzflecken vorliegen und von dem diffusen Halo bei 2,1 A leicht zu unterscheiden sind, entfällt diese Möglichkeit.

Die neuen Interferenzen sind so wenig definiert, daß eine Beziehung zu Keratinstrukturen noch schwieriger gelingt als bei den Faserinterferenzen von A stbury. Stark belichtete Keratin-Röntgenogramme in zylindrischen Röntgenkammern gleichen im Typ den Röntgenaufnahmen von Flüssigkeiten bzw. amorphen Körpern, welche sich meistens durch einen starken diffusen $D$ e b y e Ring auszeichnen, welcher für den mittleren $A b$ stand zwischen den Molekülen gilt. Solche Röntgenogramme enthalten außerdem intramolekulare Interferenzen ${ }^{4}$. Auch in unserem Falle kann die

4 Vergl. H. M a r k in K. H. M e ye r u. H. M a r k, „Hochpolymere Chemie“ Band I, Leipzig 1940. starke und diffuse Schwärzung zwischen 3,9 und 4,6 Å von der Beugung der Röntgenstrahlen an den Einzelketten des amorphen Teiles herrühren. Zahlreiche andere Körper sind so aufgebaut, daß der mittlere Abstand zwischen den Ketten etwa $4 \AA$ beträgt. $3 \div 5 \AA$ A rechnet man allgemein als ungefähren zwischenmolekularen Abstand. Die übrigen Interferenzen lassen sich als die zweite bis vierte Ordnung dieses Halos auffassen. Möglicherweise besteht ein Zusammenhang dieser Interferenzen mit intramolekularen Periodizitäten, von denen einige in der Tabelle aufgeführt sind. Eine Entscheidung zwischen diesen beiden Möglichkeiten läßt sich an Hand des bisher vorliegenden Materials nicht treffen.

Intramolekulare Periodizitäten bei Proteinen in Å.

I. Abstände.

$$
\begin{array}{ll}
\mathrm{C}-\mathrm{C} & 1,54 \\
\mathrm{C}-\mathrm{N} & 1,33 \\
\mathrm{C}-\mathrm{O} & 1,21
\end{array}
$$

II. Periodizitäten in der Kettenrichtung (Höhen).

$$
\begin{array}{ll}
\mathrm{C}-\mathrm{C} & 1,26 \\
\mathrm{CO}-\mathrm{N} & 1,09 \\
\mathrm{CHR}-\mathrm{N} & 1,14 \\
\mathrm{C}-\mathrm{N}-\mathrm{C} & 2,25
\end{array}
$$

\title{
Beiträge zur Kenntnis der Wirkungsweise des 4.4'-Dichlordiphenyl-trichlormethyl-methans beim Warmblüter
}

\author{
Von Ludwig Emmel und Martin Krüpe \\ Aus dem Robert-Koch-Institut für Infektionskrankheiten Berlin \\ (Z. Naturforschg. 1, 691-695 [1946]; eingegangen am 4. Juni 1946)
}

$\mathrm{D}^{\prime}$ ie vielseitige Verwendung des 4.4'-Dichlordiphenyl-trichlormethyl-methans (DDT) in der Schädlingsbekämpfung hat die Nachprüfung seiner etwaigen Giftigkeit für den Warmblüter dringend erforderlich gemacht. Wenn überhaupt mit diesem lipoidlöslichen, neurotropen chemischen Körper eine Vergiftung beim Warmblüter zu erwarten war, dann lag die Vermutung nahe, daß diese, ähnlich wie beim Insekt, ein äußerst interessantes Bild ergeben würde. In der Tat konnte R. D omenjoz ${ }^{1}$ frühzeitig auf die Ähnlichkeit der Vergiftungssymptome beim Warmblüter und beim Insekt hinweisen. P. L ä u g r, R. P u lver und

1 Schweiz. med. Wschr. 1944, Nr. 36, 952.
C. M ontige $1^{2}$ haben sich mit der Wirkung des DDT beim Warmblüter ebenfalls eingehend befaßt und physiologisch-chemische Untersuchungen durchgeführt. Sie konnten mit niedrigen Organkonzentrationen starke biologische Effekte erzielen. Allerdings waren hierzu massive Dosierungen bei der peroralen Verabreichung erforderlich. Die Substanz bewirkt zunächst eine Ausschüttung des Leberglykogens und eine gleichzeitige Erhöhung des Blutzuckergehalts, dem wiederum ein erhöhter Zuckerverbrauch im Organismus unter gleichzeitiger Vermehrung der Milchsäureproduktion folgt.

\footnotetext{
2 Helv. Physiol. Pharmakol. Acta 3, 3 [1945].
} 